\title{
COVID-19 and Atrial Fibrillation in Older Patients: Does Oral Anticoagulant Therapy Provide a Survival Benefit?-An Insight from the GeroCovid Registry
}

\author{
Stefano Fumagalli ${ }^{1}$ Caterina Trevisan ${ }^{2}$ Susanna Del Signore ${ }^{3}$ Giulia Pelagalli ${ }^{1}$ Stefano Volpato ${ }^{4}$ \\ Pietro Gareri $^{5}$ Enrico Mossello ${ }^{1}$ Alba Malara ${ }^{6}$ Fabio Monzani ${ }^{7}$ Alessandra Coin ${ }^{2}$ Giuseppe Bellelli ${ }^{8}$ \\ Gianluca Zia $^{3}$ Raffaele Antonelli Incalzi ${ }^{9}$ for the GeroCovid Working Group*
}

${ }^{1}$ Geriatric Intensive Care Unit and Geriatric Arrhythmia Unit, Department of Experimental and Clinical Medicine, University of Florence and AOU Careggi, Florence, Italy

${ }^{2}$ Geriatrics Division, Department of Medicine (DIMED), University of Padua, Padua, Italy

3 Bluecompanion Itd, London, United Kingdom

${ }^{4}$ Section of Internal and Cardiorespiratory Medicine, Department of Medical Science, University of Ferrara, Ferrara, Italy

${ }^{5}$ Center for Cognitive Disorders and Dementia - Catanzaro Lido, ASP Catanzaro, Catanzaro, Italy

${ }^{6}$ Scientific Committee of National Association of Third Age Residences (ANASTE) Calabria, Lamezia Terme (Catanzaro), Catanzaro, Italy
Address for correspondence Stefano Fumagalli, MD, PhD, FEHRA, FESC, FAIAC, Geriatric Intensive Care Unit and Geriatric Arrhythmia Unit, Department of Experimental and Clinical Medicine, University of Florence and AOU Careggi, Largo Brambilla, 3-50134 Florence, Italy (e-mail: stefano.fumagalli@unifi.it).

\footnotetext{
${ }^{7}$ Geriatrics Unit, Department of Clinical and Experimental Medicine, University of Pisa, Pisa, Italy

${ }^{8}$ Acute Geriatric Unit, San Gerardo Hospital, School of Medicine and Surgery, University of Milano-Bicocca, Monza, Italy

${ }^{9}$ Unit of Geriatrics, Department of Medicine, Campus Bio Medico University and Teaching Hospital, Rome, Italy
}

\section{Abstract}

Keywords

- atrial fibrillation

- COVID-19

- DOACS

- aged

- oral anticoagulants
Introduction Atrial fibrillation (AF), the most frequent arrhythmia of older patients, associates with serious thromboembolic complications and high mortality. Coronavirus disease 2019 (COVID-19) severely affects aged subjects, determining an important prothrombotic status. The aim of this study was to evaluate mortality-related factors in older AF patients with COVID-19.

Methods Between March and June 2020, we enrolled $\geq 60$ year-old in-hospital COVID19 patients $(n=806)$ in GeroCovid, a multicenter observational study promoted by the Italian Society of Gerontology and Geriatric Medicine.

Results The prevalence of AF was $21.8 \%$. In-hospital mortality was higher in the AF group ( 36.9 vs. $27.5 \%, p=0.015$ ). At admission, $51.7,10.2$, and $38.1 \%$ of AF cases were taking, respectively, oral anticoagulants (OACs), antiplatelet agents, and no antithrombotic therapy. During hospitalization, $51 \%$ patients switched to low-molecularweight heparins. AF patients who survived were younger ( $81 \pm 8$ vs. $84 \pm 7$ years; $p=0.002)$ and had a lower $\mathrm{CHA}_{2} \mathrm{DS}_{2}$-VASc score $(3.9 \pm 1.6$ vs. $4.4 \pm 1.3 ; p=0.02)$ than those who died. OAC use before (63.1 vs. $32.3 \% ; p<0.001)$ and during hospitalization ( 34.0 vs. $12.7 \% ; p=0.002$ ) was higher among survivors. At multivariable analysis, lower age, higher self-sufficiency, less severe initial COVID-19 presentation, and the use of vitamin $\mathrm{K}$ antagonists (odds ratio $[\mathrm{OR}]=0.16,95 \%$ confidence interval $[\mathrm{Cl}]: 0.03-0.84$ ) or direct OACs (OR $=0.22,95 \% \mathrm{Cl}: 0.08-0.56$ ) at admission, or the persistence of OAC

\footnotetext{
received

February 16, 2021

accepted after revision

May 5, 2021

published online

May 7, 2021
}

* Members of the GeroCovid Working Group are listed in

-Supplementary Appendix A (available in the online version).

(c) 2021. Thieme. All rights reserved. Georg Thieme Verlag KG,

Rüdigerstraße 14 ,

70469 Stuttgart, Germany
DOI https://doi.org/ 10.1055/a-1503-3875. ISSN 0340-6245. 
during hospitalization $(\mathrm{OR}=0.05,95 \% \mathrm{Cl}$ : $0.01-0.24)$, were associated with a lower chance of in-hospital death.

Conclusion AF is a prevalent and severe condition in older COVID-19 patients. Advanced age, dependency, and relevant clinical manifestations of disease characterized a worse prognosis. Preadmission and in-hospital anticoagulant therapies were positively associated with survival.

\section{Introduction}

Atrial fibrillation (AF) is the arrhythmia most frequently diagnosed in older subjects. ${ }^{1}$ In this at-risk segment of population, AF, through a higher incidence of stroke and dementia, plays a key role in causing frailty and disability, and in increasing mortality. ${ }^{2}$ These negative outcomes are mainly linked to the upregulation of thrombotic biomarkers that characterizes AF patients. ${ }^{3,4}$

Interestingly, a prothrombotic status also qualifies as a severity factor in severe acute respiratory syndrome coronavirus 2 (SARS-CoV-2) disease-2019 (COVID-19). ${ }^{5}$ Indeed, disseminated intravascular coagulation and the most important clinical manifestations of COVID-19 at a pulmonary level usually derive from complex mechanisms simultaneously involving inflammation and the coagulation cascade. ${ }^{6}$ Therefore, thrombosis and coagulopathy have a central role in the evolution of COVID-19, and their extent often drives patients' clinical prognosis. ${ }^{5}$ Accordingly, in all patients hospitalized for COVID-19 and not anticoagulated, it is recommended to start a prophylaxis with low-molecular-weight heparin (LMWH). Instead, if already started for a medical indication, therapy with oral anticoagulants (OACs) should be maintained in subjects with no or mild symptoms of COVID-19 disease, while a switch to LMWH is recommended in the case of moderate or severe clinical states. ${ }^{5,7}$

AF per se seems to be associated with a worse COVID-19 prognosis, ${ }^{8-10}$ and uncertainty exists about the effects of chronic OACs on AF patients who develop the infection, as the available evidence is inconclusive and, often, conflicting. ${ }^{11-14}$ Clarifying this issue would be of great relevance to improve the clinical management of people with AF and COVID-19.

The aim of this study was therefore to identify the factors associated with mortality in older COVID-19 patients with AF -anamnestic or diagnosed at ward admission-focusing on the role of preadmission and in-hospital OAC therapy. To this purpose, we analyzed data from the GeroCovid study-acute wards cohort-which enrolled inpatients aged $\geq 60$ years, and it is highly representative of the real-world scenario of COVID-19 at an advanced age.

\section{Methods}

\section{Study Population}

GeroCovid Observational is a multipurpose and multicenter initiative promoted by the Italian Society of Gerontology and Geriatric Medicine that aims at investigating the impact of SARS-CoV-2 pandemics in older patients in different settings of care. ${ }^{15}$ The final endpoint of GeroCovid is to provide highquality and comprehensive data, which will help to optimize COVID-19 prevention and management of patients $\geq 60$ years. Among the secondary outcomes of the project, special attention is given to study the effects of the interaction between virus infection and relevant comorbidities on mortality and other serious unfavorable events. Overall, 66 investigational sites are actively participating. By protocol, data can be collected retrospectively and prospectively in an e-Registry since March 1, 2020. The objectives of the project are specific for each setting of care. GeroCovid was registered at ClinicalTrials.gov (NCT04379440) and the participation of each center was authorized by the corresponding local ethical committee. In the acute care, in-hospital, population, we purposed to assess clinical presentation and course, and to identify the prognostic factors of the disease. The enrollment of new cases in this section of the study ended on December 31, 2020, and the follow-up will be closed on June 30, 2021.

For the present study, we included, almost exclusively in a retrospective way ( $96.3 \%$ of the whole population), the subjects enrolled during the first wave of the COVID-19 pandemic in the 16 centers participating to the "GeroCovid acute wards" cohort of the registry. ${ }^{15}$ On this basis, present analysis is to be considered retrospective.

\section{Data Collection}

For each participant, the presence of AF was derived either from the medical history or from the hospital record at ward admission. Complete information on anamnestic chronic diseases and on pharmacological therapy before and during hospitalization was obtained from the medical records. In particular, for the purpose of this study, we considered the use of OACs (vitamin $\mathrm{K}$ antagonists [VKAs] or direct OACs [DOACs]), antiplatelet agents (APLTs), or no therapy before hospital admission, and during the hospitalization (also considering the possible switch to LMWH). In accordance with admission and in-hospital treatment, patients were categorized as: no/APLT (no antithrombotic therapy or APLTs during the hospitalization), LMWH-no OAC (LMWH during the hospitalization, no anticoagulants at admission), and LMWH-OAC (LMWH during the hospitalization, anticoagulant therapy at admission).

Similarly, data on vital signs (blood pressure, heart rate) and biochemical analyses were collected at baseline. We used a 7-level scale to assess pre-COVID-19 functional status. For the aims of the study, given the distribution of the variable in our population, we categorized patients as without versus with functional limitations, by grouping levels 1 
and 2 (the patient can walk independently or using a walking stick) and levels 3 to 7 (the patient can walk using a walker; the patient can move around with a wheelchair; the patient does not move around but he is accompanied outside on the wheelchair; the patient is confined at home, mostly lying on the bed; the patient does not autonomously stand up or get in sitting position). According to the World Health Organization (WHO) classification and patients' distribution, the severity of COVID-19 at admission was ranked as mild with no oxygen support needed, mild with low-flow oxygen support needed, and severe or critical (high-flow oxygen support required, need of noninvasive or invasive mechanical ventilation, or organ support). ${ }^{16}$

\section{Statistical Analysis}

IBM SPSS version 26.0 (64-bit edition) for macOS was used for statistical analysis. Continuous variables are expressed as mean \pm standard deviation, while discrete variables as raw numbers and percentages. The comparison of continuous variables by in-hospital mortality was performed with the Student's $t$-test or the Mann-Whitney test, when appropriate. For categorical variables, we used the chi-square test.

Accordingly, age, gender, body size, functional profile, the most important clinical conditions related to AF, the other relevant comorbidities, the use of cardiovascular drugs and antithrombotic therapy, baseline vital signs, laboratory tests associated with inflammation, arterial blood gas analysis, and severity of COVID-19 were tested in relation to outcome in univariate analysis. Then, those clinically plausible and significantly associated $(p<0.05)$ with prognosis were entered into a multivariable logistic regression analysis model, provided that the percentage of missing values for each of them was not higher than $7.4 \%$ ( $n=13 / 176$; median proportion of missing values: $5.7 \%$, interquartile range [IQR]: 5.1-7.4\%). Because of the relatively low number of $\mathrm{AF}$ patients $(n=176)$, we did not perform any statistical procedure to input missing data. Also, we excluded factors showing high collinearity with others but a weaker association with mortality. To increase the stability of the estimates, the least statistically correlated variables (with $p>0.1$ as a cutoff value) were iteratively removed from the model using a backward deletion process. ${ }^{17}$ The strength of the associations between the factors identified as significant and mortality was expressed as odds ratios (ORs) and $95 \%$ confidence intervals $(95 \% \mathrm{CIs})$. For all analyses, statistical significance was set at a $p$-value $<0.05$.

\section{Results}

From March 1 to June 9, 2020, a total of 2,474 consecutive patients were included in the GeroCovid e-Registry. Of these, 806 (32.6\%) were enrolled in hospital settings (age: $78 \pm 9$ years; men: $50.7 \%$ ). In this group, the prevalence of AF at ward admission or in clinical history was $21.8 \%(n=176$; men: $51.7 \%$ ). Gender did not differ by arrhythmia presence $(p=0.799)$; patients with AF were older $(82 \pm 8$ vs. $77 \pm 9$ years; $p<0.001$ ) and with a greater prevalence of comorbidities; accordingly, their $\mathrm{CHA}_{2} \mathrm{DS}_{2}$-VASc score was higher
(4.1 \pm 1.5 vs. $3.2 \pm 1.5 ; p<0.001)$. Overall, in-hospital mortality in those with arrhythmia was $36.9 \%(n=65 / 176)$, higher than that observed in the non-AF subjects $(27.5 \%$, $n=173 / 630 ; p=0.015$ ).

Median length of stay in hospital of the AF group was 14 days (25th-75th percentile: $6-29$ days).

When evaluating the use of antithrombotic therapy at admission, only 91 (51.7\%) AF cases were anticoagulated (22 with VKAs, $12.5 \%$, and 69 with DOACs, 39.2\%), 18 (10.2\%) just received antiplatelet drugs (APLTs), and 67 (38.1\%) were neither taking antithrombotic drugs nor APLTs. No significant differences in antithrombotic therapy at baseline were observed according to functional status $(p=0.392)$. During hospitalization, 79 (44.9\%) AF subjects were prescribed LMWH. Of these, 43 (24.4\%) were previously treated with OACs and $36(20.5 \%)$ were taking APLTs or no antiplatelet/ anticoagulant therapy. In 41 (23.3\%) patients, the ongoing treatment with OACs was confirmed also during the hospitalization, while in 35 (19.9\%) cases neither OACs nor APLTs were prescribed. In the remaining 21 (11.9\%) patients, therapy was not specified. The frequency of subjects taking OACs during the hospitalization decreased in the categories with a more severe clinical condition according to the WHO classification (mild, no oxygen support needed: $51.2 \%, n=21$; mild, low-flow oxygen support needed: $34.1 \%, n=14$; severe or critical disease: $14.6 \%, n=6 ; p=0.003$ ).

\section{Predictors of Mortality in Older AF Patients}

Among AF patients, those who died during hospitalization, when compared with survivors, were older and showed a higher prevalence of heart failure and chronic obstructive pulmonary disease. Also, they had been more frequently hospitalized for a stroke and presented a higher $\mathrm{CHA}_{2} \mathrm{DS}_{2}-$ VASc score (-Table $\mathbf{1}$ ). Median length of stay in hospital was 17 days (IQR: 7-33 days) in patients who were discharged and 9 days (IQR: $6-28$ days) in those who died $(p=0.086$ ).

Among nonsurvivors, we observed a worse pre-COVID-19 functional status and a more severe disease picture at admission, according to the WHO classification. As regards to vital signs and biochemical parameters at baseline, AF patients who died had higher values of heart rate, white blood cells, and C-reactive protein. Instead, their lymphocyte count was lower than in survivors, as were $\mathrm{PaO}_{2}$ and the ratio between $\mathrm{PaO}_{2}$ and $\mathrm{FiO}_{2}$ (-Table $\mathbf{1}$ ).

When analyzing preadmission therapy, we did not find significant differences in the use of $\beta$-blockers and statins between AF patients who died and those who survived, while treatment with renin-angiotensin system antagonists was more frequent in the latter group. The use of OACs before hospitalization, particularly DOACs, was higher in patients who survived, while antiplatelet therapy was more common in those who died (-Fig. 1). Taking into consideration inhospital therapy, survival was higher in those who were treated with OACs and LMWH-OAC (- Fig. 2).

In the first multivariable logistic regression analysis (-Table 2), older AF patients with COVID-19 who had a more advanced age, a preinfection lower functional profile, and a more severe clinical presentation of disease at 
Table 1 Clinical and laboratory characteristics of AF patients by vital status

\begin{tabular}{|c|c|c|c|}
\hline & \multicolumn{2}{|c|}{ In-hospital mortality } & \multirow[b]{2}{*}{$p$} \\
\hline & No & Yes & \\
\hline Age $(y)$ & $81 \pm 8$ & $84 \pm 7$ & 0.002 \\
\hline Women $(n, \%)$ & $56(50.5)$ & $29(44.6)$ & 0.532 \\
\hline $\begin{array}{l}\text { Body mass } \\
\text { index }\left(\mathrm{kg} / \mathrm{m}^{2}\right)\end{array}$ & $25.7 \pm 4.7$ & $24.9 \pm 6.5$ & 0.601 \\
\hline $\begin{array}{l}\text { Functional } \\
\text { limitations ( } n, \%)\end{array}$ & 39 (37.9) & $41(68.3)$ & $<0.001$ \\
\hline Arthrosis (n, \%) & $37(34.9)$ & $13(23.2)$ & 0.154 \\
\hline $\begin{array}{l}\text { Cardiac } \\
\text { diseases }(n, \%)\end{array}$ & $66(62.9)$ & $46(73.0)$ & 0.237 \\
\hline $\mathrm{CHF}(n, \%)$ & $29(27.4)$ & $26(42.6)$ & 0.043 \\
\hline CKD $(n, \%)$ & $27(25.5)$ & $9(14.8)$ & 0.121 \\
\hline $\operatorname{COPD}(n, \%)$ & $18(17.1)$ & $19(31.7)$ & 0.035 \\
\hline Depression ( $n, \%)$ & $12(11.4)$ & $6(10.3)$ & 1.000 \\
\hline Diabetes $(n, \%)$ & $35(32.7)$ & $23(36.5)$ & 0.620 \\
\hline $\begin{array}{l}\text { Hepatic } \\
\text { diseases }(n, \%)\end{array}$ & $2(1.9)$ & $1(1.6)$ & 1.000 \\
\hline Hypertension (n, \%) & $81(75.0)$ & $44(72.1)$ & 0.717 \\
\hline Obesity (n, \%) & $18(17.5)$ & $11(19.0)$ & 0.833 \\
\hline $\begin{array}{l}\text { Peripheral artery } \\
\text { disease }(n, \%)\end{array}$ & $21(20.8)$ & $16(27.6)$ & 0.337 \\
\hline Stroke $(n, \%)$ & $10(9.6)$ & $13(21.0)$ & 0.041 \\
\hline $\begin{array}{l}\mathrm{CHA}_{2} \mathrm{DS}_{2} \text {-VASs } \\
\text { (score) }\end{array}$ & $3.9 \pm 1.6$ & $4.4 \pm 1.3$ & 0.020 \\
\hline $\begin{array}{l}\text { Anti-arrhythmic } \\
\text { drugs }(n, \%)\end{array}$ & $20(18.0)$ & $1(1.5)$ & 0.001 \\
\hline Beta-blockers ( $n, \%)$ & $55(49.5)$ & $27(41.5)$ & 0.349 \\
\hline Digitalis ( $n, \%)$ & $8(7.2)$ & $6(9.2)$ & 0.774 \\
\hline Diuretics ( $n, \%)$ & 41 (36.9) & $24(36.9)$ & 1.000 \\
\hline $\operatorname{RAAS}(n, \%)$ & $51(45.9)$ & $18(27.7)$ & 0.025 \\
\hline Statins (n, \%) & $28(25.2)$ & $10(15.4)$ & 0.135 \\
\hline $\begin{array}{l}\text { WHO disease } \\
\text { severity }(n, \%)\end{array}$ & & & $<0.001$ \\
\hline Mild, no $\mathrm{O}_{2}$ support & $41(38.7)$ & $9(14.3)$ & \\
\hline Mild, $\mathrm{O}_{2}$ support & $51(48.1)$ & $26(41.3)$ & \\
\hline Severe/critical & $14(13.2)$ & $28(44.4)$ & \\
\hline Heart rate (bpm) & $79 \pm 16$ & $87 \pm 17$ & 0.003 \\
\hline $\begin{array}{l}\text { Systolic arterial } \\
\text { pressure }(\mathrm{mmHg})\end{array}$ & $129 \pm 22$ & $123 \pm 26$ & 0.121 \\
\hline $\begin{array}{l}\text { Diastolic arterial } \\
\text { pressure }(\mathrm{mmHg})\end{array}$ & $73 \pm 12$ & $71 \pm 15$ & 0.427 \\
\hline $\mathrm{pH}$ & $7.44 \pm 0.05$ & $7.46 \pm 0.07$ & 0.255 \\
\hline $\mathrm{PaO}_{2}(\mathrm{mmHg})$ & $84 \pm 40$ & $72 \pm 34$ & 0.011 \\
\hline $\mathrm{PaCO}_{2}(\mathrm{mmHg})$ & $39 \pm 12$ & $38 \pm 13$ & 0.593 \\
\hline $\mathrm{HCO}_{3}{ }^{-}(\mathrm{mEq} / \mathrm{L})$ & $25.4 \pm 3.6$ & $26.8 \pm 4.9$ & 0.170 \\
\hline $\mathrm{FiO}_{2}(\%)$ & $34.8 \pm 24.0$ & $41.6 \pm 25.5$ & 0.059 \\
\hline
\end{tabular}

Table 1 (Continued)

\begin{tabular}{|l|l|l|l|}
\hline & \multicolumn{2}{l|}{ In-hospital mortality } & \\
\hline & No & Yes & $p$ \\
\hline $\mathrm{PaO}_{2} / \mathrm{FiO}_{2}$ & $286 \pm 130$ & $215 \pm 108$ & 0.001 \\
\hline Hemoglobin $(\mathrm{g} / \mathrm{dL})$ & $12.1 \pm 1.8$ & $11.9 \pm 2.3$ & 0.479 \\
\hline Platelets $\left(n \times 10^{9} / \mathrm{L}\right)$ & $214 \pm 89$ & $217 \pm 98$ & 0.815 \\
\hline WBC $\left(n \times 10^{9} / \mathrm{L}\right)$ & $7.47 \pm 4.89$ & $9.14 \pm 4.74$ & 0.006 \\
\hline Lymphocytes $(\%)$ & $16.7 \pm 10.4$ & $10.3 \pm 6.5$ & 0.001 \\
\hline LDH $(\mathrm{U} / \mathrm{L})$ & $337 \pm 204$ & $438 \pm 270$ & 0.066 \\
\hline $\mathrm{CRP}(\mathrm{mg} / \mathrm{L})$ & $64 \pm 77$ & $144 \pm 260$ & 0.036 \\
\hline INR & $2.1 \pm 3.0$ & $1.9 \pm 2.2$ & 0.775 \\
\hline
\end{tabular}

Abbreviations: $\mathrm{CHF}$, presence of signs and symptoms of heart failure; CKD, chronic kidney disease; COPD, chronic obstructive pulmonary disease; CRP, C-reactive protein; $\mathrm{FiO}_{2}$, inspired oxygen fraction; Functional limitations, the patient needs a walker or a wheelchair to move, or he is bed-ridden; INR, international normalized ratio; LDH, lactate dehydrogenase; Mild, $\mathrm{O}_{2}$ support, mild disease with low-flow oxygen support needed; RAAS, renin-angiotensin-aldosterone system antagonists; Severe/critical, disease needing high-flow oxygen support, noninvasive or invasive mechanical ventilation, or organ support; WBC, white blood cell count; WHO, World Health Organization classification of severity of COVID-19.

Note: Please note that for some variables raw numbers may be associated with slightly different percentages due to the presence of missing values.

admission showed a higher risk of in-hospital mortality. The predisease use of OACs, both VKAs and DOACs, when compared with the use of APLTs or none, was associated with lower odds of in-hospital mortality. This effect was similar for DOACs and VKAs (OR=0.74, 95\% CI: 0.13-4.17, $p=0.734$, considering as exposure DOACs vs. VKAs). No significant associations with prognosis were observed for heart rate, history of heart failure, chronic obstructive pulmonary disease, stroke, and the use of renin-angiotensin system antagonists (-Table 2). In the second multivariable logistic regression analysis, considering in-hospital antithrombotic therapy, OACs and LMWH-OAC were associated with a lower

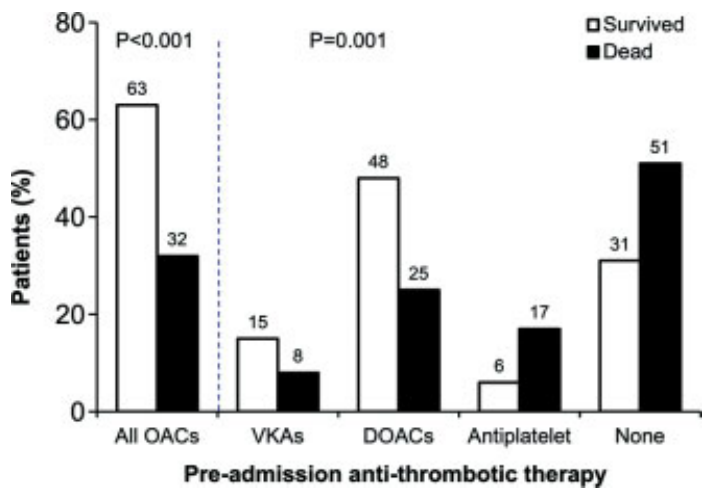

Fig. 1 In-hospital vital status in AF patients with COVID-19 by use of antiplatelet/anticoagulant agents at ward admission. AF, atrial fibrillation; All OACs, VKAs and DOACs; DOACs, direct oral anticoagulants; OAC, oral anticoagulant; VKA, vitamin $\mathrm{K}$ antagonist. 


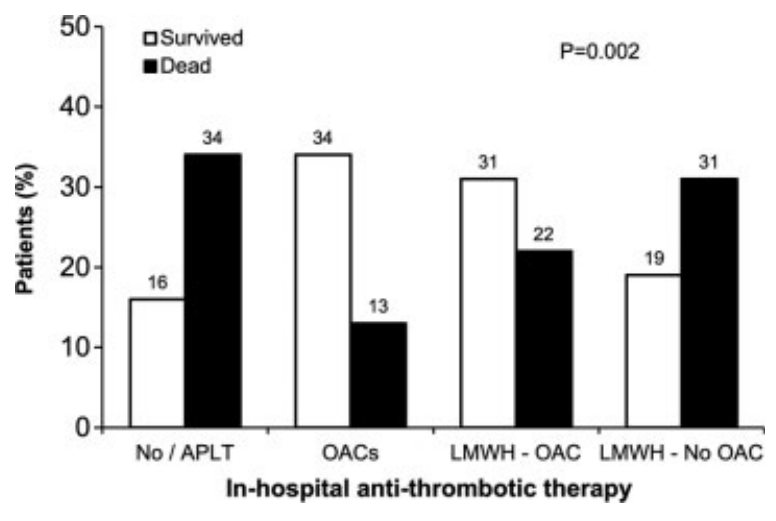

Fig. 2 In-hospital vital status in AF patients with COVID-19 by use of antithrombotic agents during hospitalization. No/APLT, no antithrombotic therapy or use of APLTs during the hospitalization; LMWHno OAC: therapy with LMWHs during the hospitalization, no anticoagulants at admission; LMWH-OAC: therapy with LMWHs during the hospitalization, anticoagulant therapy at admission. AF, atrial fibrillation; APLTs, antiplatelet agents; LMWH, low-molecular-weight heparin; OAC, oral anticoagulant.

risk of mortality than that observed in subjects receiving APLTs or no therapy at all (-Table 3 ).

\section{Discussion}

The results of the present analysis from the GeroCovid initiative demonstrate that many of the older patients hospitalized for COVID-19-in our study $>20 \%$-could present AF at ward admission or in her/his clinical history. The arrhythmia was associated with a mortality rate approaching $40 \%$, in proportion $>30 \%$ higher than that observed in individuals without AF. Among AF patients with COVID-19, increasing age, a lower functional profile, and a more severe clinical status at baseline were related to higher in-hospital mortali- ty. On the other hand, the preadmission use of OACs-either VKAs or DOACs-was associated with a significantly higher survival. Also, in-hospital prescription of oral anticoagulation and LMWH in those previously treated with OACs was more common in subjects who survived.

The observed prevalence of AF is higher than that found in a specialized U.S. center (15.8\%), in which, however, population was younger than ours. ${ }^{18}$

Among our patients, age was independently associated with mortality. In an Italian experience, when compared with the $<62$ year-old group, subjects aged $\geq 75$ and 62 to 74 years had a risk of dying 8 and 3 times higher, respectively, even after adjustment for number of comorbidities, respiratory rate and function, renal failure, and number of platelets. ${ }^{19}$ In the GeroCovid population, the association with age persisted after the adjustment for functional profile. Impaired functional capabilities rather than individual diseases characterized AF patients with a worse prognosis. In line with our findings, previous works showed that factors like history of falls and frailty were associated with both risk and severity of COVID-19 at older ages. ${ }^{20,21}$ In 375 consecutive patients younger than ours (mean age: 66 years), disability in basic activities of daily living was a significant predictor of mortality even after adjustment for age, gender, and the presence of comorbidities. Polypharmacy and the quick Sequential Organ Failure Assessment score, an index of disease severity, did not correlate at all with outcome. Importantly, the effect on survival of a low physical performance status was similar to that observed for an age increase of 15 years. Accordingly, age and declining physical function variably concur to shape the profile of risk, even if the importance of other factors cannot be excluded. ${ }^{22}$

Renin-angiotensin system antagonist therapy was not associated with an increased mortality in our population. This is an important finding, because the renin-angiotensin

Table 2 Variables associated with mortality in patients with AF and COVID-19 taking into consideration preadmission oral anticoagulant (OAC) therapy ${ }^{\mathrm{a}}$

\begin{tabular}{|l|l|l|l|l|}
\hline & $\beta \pm$ es & $p$ & OR & $95 \% \mathrm{Cl}$ \\
\hline Age $(\Delta$.year) & $0.07 \pm 0.03$ & 0.042 & 1.07 & $(1.00-1.14)$ \\
\hline Functional limitations (yes vs. no) & $1.40 \pm 0.52$ & 0.007 & 4.04 & $(1.47-11.11)$ \\
\hline COPD (yes vs. no) & $1.02 \pm 0.53$ & 0.056 & 2.76 & $(0.97-7.86)$ \\
\hline OACs at ward admission & 1 & 0.002 & 1 & $/$ \\
\hline VKAs vs. no OACs & $-1.83 \pm 0.85$ & 0.031 & 0.16 & $(0.03-0.84)$ \\
\hline DOACs vs. no OACs & $-1.53 \pm 0.49$ & 0.002 & 0.22 & $(0.08-0.56)$ \\
\hline WHO disease severity & & $<0.001$ & & \\
\hline Mild, $\mathrm{O}_{2}$ support vs. mild, no $\mathrm{O}_{2}$ support & $0.58 \pm 0.55$ & 0.298 & 1.78 & $(0.60-5.28)$ \\
\hline Severe/critical vs. mild, no $\mathrm{O}_{2}$ support & $2.44 \pm 0.68$ & $<0.001$ & 11.53 & $(3.02-43.98)$ \\
\hline
\end{tabular}

Abbreviations: AF, atrial fibrillation; $\mathrm{CHF}$, presence of signs and symptoms of heart failure; $\mathrm{Cl}$, confidence interval; COPD, chronic obstructive pulmonary disease; DOACs, direct OACs; Functional limitations, the patient needs a walker or a wheelchair to move, or he is bed-ridden; Mild, $\mathrm{O}_{2}$ support, mild disease with low-flow oxygen support needed; OAC, oral anticoagulant; RAAS, renin-angiotensin-aldosterone system antagonists; Severe/critical, disease needing high-flow oxygen support, noninvasive or invasive mechanical ventilation, or organ support; VKAs, vitamin K antagonists; WHO, World Health Organization classification of severity of COVID-19.

Note: Variables backward deleted from the model: $\operatorname{CHF}(p=0.119)$; heart rate $(p=0.221)$; $\operatorname{RAAS}(p=0.119)$; stroke $(p=0.565)$.

${ }^{\mathrm{a}}$ Results derive from multivariable logistic regression analysis (overall predictivity: $76.5 \% ; p<0.001$ ). 
Table 3 Variables associated with mortality in patients with AF and COVID-19 taking into consideration antithrombotic therapy during hospitalization ${ }^{\mathrm{a}}$

\begin{tabular}{|l|l|l|l|l|}
\hline & $\boldsymbol{\beta} \pm$ es & $p$ & OR & $95 \% \mathrm{Cl}$ \\
\hline Age $(\Delta$.year) & $0.07 \pm 0.03$ & 0.047 & 1.07 & $(1.00-1.14)$ \\
\hline Functional limitations (yes vs. no) & $1.56 \pm 0.57$ & 0.006 & 4.78 & $(1.56-14.68)$ \\
\hline CHF (yes vs. no) & $1.00 \pm 0.53$ & 0.062 & 2.71 & $(0.95-7.72)$ \\
\hline Antithrombotic therapy & 1 & $<0.001$ & $l$ & $/$ \\
\hline OAC vs. no/APLT & $-3.04 \pm 0.82$ & $<0.001$ & 0.05 & $(0.01-0.24)$ \\
\hline LMWH-no OAC vs. no/APLT & $-0.81 \pm 0.70$ & 0.246 & 0.45 & $(0.11-1.74)$ \\
\hline LMWH-OAC vs. no/APLT & $-2.01 \pm 0.68$ & 0.003 & 0.13 & $(0.03-0.51)$ \\
\hline WHO disease severity & & 0.001 & & \\
\hline Mild, $\mathrm{O}_{2}$ support vs. mild, no $\mathrm{O}_{2}$ support & $0.34 \pm 0.64$ & 0.593 & 1.41 & $(0.40-4.97)$ \\
\hline Severe/critical vs. mild, no $\mathrm{O}_{2}$ support & $2.37 \pm 0.75$ & 0.002 & 10.69 & $(2.45-46.72)$ \\
\hline
\end{tabular}

Abbreviations: AF, atrial fibrillation; APLT, antiplatelet agent; CHF, presence of signs and symptoms of heart failure; COPD, chronic obstructive pulmonary disease; Functional limitations, the patient needs a walker or a wheelchair to move, or he is bed-ridden; LMWH, low-molecular-weight heparin; LMWH-no OAC, LMWH during the hospitalization, no anticoagulants at admission; LMWH-OAC, LMWH during the hospitalization, anticoagulant therapy at admission; Mild, $\mathrm{O}_{2}$ support, mild disease with low-flow oxygen support needed; No/APLT, no antithrombotic therapy or use of antiplatelet agents during the hospitalization; OAC, oral anticoagulant; RAAS, renin-angiotensin-aldosterone system antagonists; Severe/critical, disease needing high-flow oxygen support, noninvasive or invasive mechanical ventilation, or organ support; WHO, World Health Organization classification of severity of COVID-19.

Note: Variables backward deleted from the model: $\operatorname{COPD}(p=0.149)$; heart rate $(p=0.214)$; $\operatorname{RAAS}(p=0.353)$; stroke $(p=0.325)$.

${ }^{a}$ Results derive from a multivariable logistic regression analysis (overall predictivity: $79.4 \% ; p<0.001$ ).

system is upregulated when the arrhythmia is present, and angiotensin-converting enzyme (ACE) inhibitors and angiotensin II receptor blockers effectively prevent $\mathrm{AF}$ onset in subjects with hypertension and left ventricular dysfunction. ${ }^{1}$ Some evidence suggests that the SARS-CoV-2 can interact with the ACE2 receptors on pericytes and perivascular cells in lungs and myocardium, leading, through an alteration in tissue permeability, to changes in extracellular fluid content, inflammation, and tissue fibrosis. These modifications could determine the development of $\mathrm{AF}^{23}$ Importantly, to perpetuate a vicious cycle, ACE2 expression is increased by the presence of the arrhythmia itself. ${ }^{24}$ In accordance with our findings, the use of renin-angiotensin system antagonists did not result in an increase of hospitalizations, need for intensive care, and mortality for COVID-19 in a recent study involving a population slightly younger than ours. ${ }^{25}$

The most interesting result of our analysis was the association between the use of OACs at ward admission and lower in-hospital mortality. In our patients, in line with guideline recommendations, DOACs were more frequently used than VKAs. ${ }^{1}$ However, the overall proportion of treated older AF patients, as found in everyday clinical practice, ${ }^{26}$ was still low, slightly higher than $50 \%$, a rate found also in U.S. seniors with $\mathrm{AF}^{27}$

As previously reported, conflicting evidence exists about the benefits of OACs in COVID-19 patients. In a small polycentric Italian experience, the preadmission use of anticoagulants did not result in a higher in-hospital survival rate of subjects with SARS-CoV-2 infection. ${ }^{11}$ In a Swedish nationwide registry analysis, the use of DOACs in AF patients, when compared with nonuse in both AF and cardiovascular sub- jects, did not result in a lower rate of hospitalizations and of the composite outcome of admissions and death in intensive care unit. Worthy of note, the mean age of the studied population was lower (73.6 years) than that observed in the GeroCovid database, and in a sensitivity analysis, allcause mortality was reduced in the DOAC cohort. ${ }^{14}$ In the HOPE Registry, enrolling COVID-19 patients in South America and Europe, oral anticoagulation at baseline, prescribed for AF, deep vein thrombosis, or pulmonary embolism, was correlated with a significantly lower survival. The presence of comorbidities and the development of respiratory failure or systemic inflammatory response syndrome greatly compromised the outcome of treated patients. ${ }^{12}$ In a sample of 4,389 AF patients enrolled in New York during the spring pandemic, both the use of a prophylactic and a therapeutic regimen of anticoagulation were associated with lower inhospital mortality. ${ }^{13}$ Finally, in line with our results, a French experience involving 2,878 COVID-19 patients (mean age: 67 years) found that prehospital oral anticoagulation, prescribed for both AF (68.1\%) and venous thromboembolism or other conditions (31.9\%), was correlated to a reduced risk of intensive care unit admission and/or death. Interestingly, no benefit was observed for therapeutic or prophylactic treatment started in hospital. ${ }^{28}$

COVID-19 patients show frequent alterations of the coagulation system characterized by higher levels of D-dimer, reduced platelet count, and prolonged prothrombin time. More relevant changes seem to characterize subjects with a worse prognosis. AF may amplify these alterations. Indeed, it was observed that the incidence of thromboembolic events considerably increased in patients who developed $\mathrm{AF}$ in the 
course of SARS-CoV-2 infection. ${ }^{29}$ It must be considered that during COVID-19, venous and arterial thrombosis, thrombotic microangiopathy, and disseminated intravascular coagulation are significant determinants of a worse prognosis. All these phenomena are variably related to alterations in the coagulation system, platelet function, and endothelium, and collectively contribute to the COVID-19-related thromboinflammatory status. Age, diabetes, obesity, and cardiovascular risk factors can exacerbate these alterations, including the inflammatory response to the infection, and may lead to further increases in patients' morbidity and mortality. 6,30 Therefore, because of the COVID-19 association with a hypercoagulable state, our findings let us hypothesize an additional possible benefit of chronic anticoagulant therapy in AF patients. The results of some trials that are investigating the usefulness of anticoagulation for prophylaxis in ambulatory or low-risk patients ${ }^{5}$ will further contribute to verify such a hypothesis.

\section{Study Limitations and Strengths}

This study has some limitations. Given that our patients were $\geq 60$ years, with a mean age of 82 years, our conclusions may not be generalized to a younger COVID-19 population. Concerning ethnicity, almost all our patients were Caucasian, a finding in line with present composition of Italian society and the age criteria for enrollment in GeroCovid. Moreover, information on socioeconomic status was not available; therefore, we could not evaluate this factor in our analyses. However, in the majority of cases, enrolled subjects were retired, and it is to be considered that the Italian health system is completely run by the Government, a circumstance mitigating the effects of economic status on acute care medical assistance. We must also mention that our results were obtained analyzing hospitalized patients. Extrapolating our findings to subjects assisted in different settings of care, such as the ambulatory or the nursing home, could bring to inappropriate assumptions. We did not consider incident AF, but our attention was focused on the presence of AF at ward admission and on anticoagulant therapy. Furthermore, we were not able to differentiate the patterns of the arrhythmia (i.e., first diagnosed, paroxysmal, persistent, long-standing persistent, and permanent). However, because of advanced age and the high $\mathrm{CHA}_{2} \mathrm{DS}_{2}$-VASc score, the indication to oral anticoagulation was mandatory in almost all cases. Due to the high proportion of patients switching their in-hospital antithrombotic treatment to LMWHs, it was impossible to separately analyze the effects on survival of VKAs or DOACs, and of APLTs and no therapy. Furthermore, we did not differentiate between the prophylactic and the therapeutic dose of LMWHs. However, it can be hypothesized that subjects who were treated with OACs prior to admission received a higher in-hospital dose of the drugs. Because of the severe clinical conditions of COVID-19-hospitalized older subjects, their isolation, and the possibility of medical and nursing staff to contact families only by phone, it was impossible to perform a complete geriatric multidimensional evaluation. Therefore, we cannot rule out that the inclusion in our models of other patients' health aspects, such as neurocognitive profile, physical performance, and affective symptoms, could have influenced our results. The findings of the present analysis derive from an observational registry. For this reason, our results do not allow to conclusively affirm a cause-effect relationship. On the other hand, strengths of our work lie in the enrolled patients being highly representative of the real-life older COVID-19 population as well as in the large set of anamnestic, clinical, and biochemical data collected. We did not observe differences in OACs' prescription by functional status at baseline. This finding strengthens our conclusions about the association between oral anticoagulation use and improved in-hospital survival, excluding a possible bias in therapy selection and prognosis linked to a pre-existing condition of disability and frailty. Last, the research activities implemented by the GeroCovid initiative, including weekly meetings of the scientific committee and frequent education sessions for the researchers involved in the project, minimize the risk of bias due to the multicenter nature of the study.

In conclusion, the results of this study show that $\mathrm{AF}$ is a highly prevalent condition in older patients hospitalized for COVID-19. The arrhythmia might amplify the effects of the SARS-CoV-2, further modifying the inflammation cascade and the coagulation system, especially in the frailest patients. Importantly, both oral anticoagulation therapy and the in-hospital switch from OACs to LMWHs are associated with a greater chance of survival. These findings suggest that the use of an effective antithrombotic therapy in the early phases of disease could positively impact the prognosis of COVID-19 older patients.

\section{What is known about this topic?}

- Atrial fibrillation is a frequent condition in older patients.

- COVID-19 has a severe prognosis at an advanced age.

- Atrial fibrillation is associated with a worse COVID-19 course.

- Both atrial fibrillation and COVID-19 exert a significant influence on coagulation cascade.

\section{What does this paper add?}

- The use of preadmission and in-hospital oral anticoagulation in older patients with atrial fibrillation and COVID-19 correlates with improved survival.

- In-hospital low-molecular-weight heparin therapy, when following oral anticoagulation at home, is associated with reduced mortality in older patients with atrial fibrillation and COVID-19.

- A better preadmission functional profile is related to higher survival in an elderly population with atrial fibrillation and COVID-19.

Conflict of Interest

None declared. 


\section{References}

1 Hindricks G, Potpara T, Dagres N, et al; ESC Scientific Document Group. 2020 ESC Guidelines for the diagnosis and management of atrial fibrillation developed in collaboration with the European Association for Cardio-Thoracic Surgery (EACTS). Eur Heart J 2021;42(05):373-498

2 Fumagalli S, Potpara TS, Bjerregaard Larsen T, et al. Frailty syndrome: an emerging clinical problem in the everyday management of clinical arrhythmias. The results of the European Heart Rhythm Association survey. Europace 2017;19:1896-1902

3 Liles J, Liles J, Wanderling C, Syed M, Hoppensteadt D, Fareed J. Increased level of thrombotic biomarkers in patients with atrial fibrillation despite traditional and new anticoagulant therapy. Clin Appl Thromb Hemost 2016;22(08):743-748

4 Kim D, Yang PS, Yu HT, et al. Risk of dementia in stroke-free patients diagnosed with atrial fibrillation: data from a population-based cohort. Eur Heart J 2019;40(28):2313-2323

5 Ortega-Paz L, Capodanno D, Montalescot G, Angiolillo DJ. Coronavirus disease 2019-associated thrombosis and coagulopathy: review of the pathophysiological characteristics and implications for antithrombotic management. J Am Heart Assoc 2021;10(03): e019650

6 Gu SX, Tyagi T, Jain K, et al. Thrombocytopathy and endotheliopathy: crucial contributors to COVID-19 thromboinflammation. Nat Rev Cardiol 2021;18(03):194-209

7 Bikdeli B, Madhavan MV, Jimenez D, et al; Global COVID-19 Thrombosis Collaborative Group, Endorsed by the ISTH, NATF, ESVM, and the IUA, Supported by the ESC Working Group on Pulmonary Circulation and Right Ventricular Function. COVID-19 and thrombotic or thromboembolic disease: implications for prevention, antithrombotic therapy, and follow-up: JACC stateof-the-art review. J Am Coll Cardiol 2020;75(23):2950-2973

8 Inciardi RM, Adamo M, Lupi L, Metra M. Atrial fibrillation in the COVID-19 era: simple bystander or marker of increased risk? Eur Heart J 2020;41(32):3094

9 Fumagalli S, Salani B, Gabbani L, Mossello E, Ungar A. Covid-19 cases in a no-Covid-19 geriatric acute care setting. A sporadic occurrence? Eur J Intern Med 2020;77:141-142

10 Mountantonakis SE, Saleh M, Fishbein J, et al; Northwell COVID19 Research Consortium. Atrial fibrillation is an independent predictor for in-hospital mortality in patients admitted with SARS-CoV-2 infection. Heart Rhythm 2021;18(04):501-507

11 Russo V, Di Maio M, Attena E, et al. Clinical impact of preadmission antithrombotic therapy in hospitalized patients with COVID-19: a multicenter observational study. Pharmacol Res 2020;159:104965

12 Rivera-Caravaca JM, Núñez-Gil IJ, Vivas D, et al; HOPE COVID-19 Investigators. Clinical profile and prognosis in patients on oral anticoagulation before admission for COVID-19. Eur J Clin Invest 2021;51(01):e13436

13 Nadkarni GN, Lala A, Bagiella E, et al. Anticoagulation, bleeding, mortality, and pathology in hospitalized patients with COVID-19. J Am Coll Cardiol 2020;76(16):1815-1826

14 Flam B, Wintzell V, Ludvigsson JF, Mårtensson J, Pasternak B. Direct oral anticoagulant use and risk of severe COVID-19. J Intern Med 2021;289(03):411-419
15 Trevisan C, Del Signore S, Fumagalli S, et al; GeroCovid Working Group. Assessing the impact of COVID-19 on the health of geriatric patients: the European GeroCovid Observational Study. Eur J Intern Med 2021;87:29-35

16 WHO Novel Coronavirus. COVID-19 Therapeutic Trial Synopsis. In: WHO. ed. WHO R\&D Blueprint. Geneva: World Health Organization; 2020:1-12

17 LaValley MP. Logistic regression. Circulation 2008;117(18): 2395-2399

18 Peltzer B, Manocha KK, Ying X, et al. Outcomes and mortality associated with atrial arrhythmias among patients hospitalized with COVID-19. J Cardiovasc Electrophysiol 2020;31(12): 3077-3085

19 Fumagalli C, Rozzini R, Vannini M, et al. Clinical risk score to predict in-hospital mortality in COVID-19 patients: a retrospective cohort study. BMJ Open 2020;10(09):e040729

20 Sacco G, Foucault G, Briere O, Annweiler C. COVID-19 in seniors: findings and lessons from mass screening in a nursing home. Maturitas 2020;141:46-52

21 Petermann-Rocha F, Hanlon P, Gray SR, et al. Comparison of two different frailty measurements and risk of hospitalisation or death from COVID-19: findings from UK Biobank. BMC Med 2020;18(01):355

22 Laosa O, Pedraza L, Álvarez-Bustos A, et al. Rapid assessment at hospital admission of mortality risk from COVID-19: the role of functional status. J Am Med Dir Assoc 2020;21:1798.e2-1802.e2

23 Stone E, Kiat H, McLachlan CS. Atrial fibrillation in COVID-19: a review of possible mechanisms. FASEB J 2020;34(09): 11347-11354

24 Sanchis-Gomar F, Perez-Quilis C, Lavie CJ. Should atrial fibrillation be considered a cardiovascular risk factor for a worse prognosis in COVID-19 patients? Eur Heart J 2020;41(32):3092-3093

25 de Abajo FJ, Rodríguez-Martín S, Lerma V, et al; MED-ACE2COVID19 study group. Use of renin-angiotensin-aldosterone system inhibitors and risk of COVID-19 requiring admission to hospital: a case-population study. Lancet 2020;395(10238):1705-1714

26 Fumagalli S, Said SAM, Laroche C, et al; EORP-AF Investigators. Age-related differences in presentation, treatment, and outcome of patients with atrial fibrillation in Europe: the EORP-AF general pilot registry (EURObservational Research Programme-Atrial Fibrillation). JACC Clin Electrophysiol 2015;1(04):326-334

27 Fohtung RB, Novak E, Rich MW. Effect of new oral anticoagulants on prescribing practices for atrial fibrillation in older adults. J Am Geriatr Soc 2017;65(11):2405-2412

28 Chocron R, Galand V, Cellier J, et al. Anticoagulation before hospitalization is a potential protective factor for COVID-19: insight from a French multicenter cohort study. J Am Heart Assoc 2021;10(08):e018624

29 Pardo Sanz A, Salido Tahoces L, Ortega Pérez R, González Ferrer E, Sánchez Recalde Á, Zamorano Gómez JL. New-onset atrial fibrillation during COVID-19 infection predicts poor prognosis. Cardiol J $2021 ; 28(01): 34-40$

30 Fumagalli S, Said SA, Laroche C, et al; EORP-AF General Pilot Registry Investigators. Management and prognosis of atrial fibrillation in diabetic patients: an EORP-AF General Pilot Registry report. Eur Heart J Cardiovasc Pharmacother 2018;4(03):172-179 